Louisiana State University

LSU Digital Commons

Faculty Publications

Department of Biological Sciences

$1-1-1998$

\title{
Crystal structure of apo-glycine N-methyltransferase (GNMT)
}

R. Pattanayek

Vanderbilt University School of Medicine

M. E. Newcomer

Vanderbilt University School of Medicine

C. Wagner

Vanderbilt University School of Medicine

Follow this and additional works at: https://digitalcommons.Isu.edu/biosci_pubs

\section{Recommended Citation}

Pattanayek, R., Newcomer, M., \& Wagner, C. (1998). Crystal structure of apo-glycine N-methyltransferase (GNMT). Protein Science, 7 (6), 1326-1331. https://doi.org/10.1002/pro.5560070608

This Article is brought to you for free and open access by the Department of Biological Sciences at LSU Digital Commons. It has been accepted for inclusion in Faculty Publications by an authorized administrator of LSU Digital Commons. For more information, please contact ir@lsu.edu. 


\title{
Crystal structure of apo-glycine $\mathrm{N}$-methyltransferase (GNMT)
}

\author{
R. PATTANAYEK, ${ }^{1}$ M.E. NEWCOMER, ${ }^{1}$ AND C. WAGNER ${ }^{1,2}$ \\ ${ }^{1}$ Department of Biochemistry, Vanderbilt University School of Medicine, Nashville, Tennessee 37232-0146 \\ ${ }^{2}$ Veterans Administration Medical Center, Nashville, Tennessee 37232-0146
}

(ReCEIVED November 24, 1997; ACCEPTED March 18, 1998)

\begin{abstract}
The crystal structure of the recombinant apo-form of glycine N-methyltransferase (GNMT) has been determined at $2.5 \AA$ resolution. GNMT is a tetrameric enzyme (monomer $M_{\mathrm{r}}=32,423 D_{a}, 292$ amino acids) that catalyzes the transfer of a methyl group from S-adenosylmethionine (AdoMet) to glycine with the formation of S-adenosylhomocysteine (AdoHcy) and sarcosine (N-methylglycine). GNMT is a regulatory enzyme, which is inhibited by 5-methyltetrahydrofolate pentaglutamate and believed to control the ratio of AdoMet to AdoHcy in tissues. The crystals belong to the orthorhombic space group $\mathrm{P}_{1} 2_{1} 2(a=85.39, b=174.21, c=44.71 \AA)$ and contain one dimer per asymmetric unit. The AdoMet-GNMT structure served as the starting model. The structure was refined to an $R$-factor of $21.9 \%$. Each monomer is a three-domain structure with a large cavity enclosed by the three domains. The tetramer resembles a square with a central channel about which $\mathrm{N}$-terminal domains are intertwined. Only localized changes of the residues involved in the binding pocket are observed for the apo-GNMT structure when compared to that determined in the presence of substrate and substrate analog.
\end{abstract}

Keywords: folate binding protein; glycine N-methyltransferase; protein structure

Glycine N-methyltransferase is an abundant enzyme in liver where it makes up about $1-3 \%$ of the soluble protein in the cytosol (Heady \& Kerr, 1973). It catalyzes the transfer of a methyl group from S-adenosylmethionine (AdoMet) to glycine with the formation of S-adenosylhomocysteine (AdoHcy) and sarcosine $(\mathrm{N}$ methylglycine). Sarcosine has no known physiological role and it has been suggested that the function of GNMT is not to synthesize sarcosine, but rather to control the ratio of AdoMet to AdoHcy (Ogawa \& Fujioka, 1982). The cDNA and genomic DNA for rat liver GNMT have been cloned and sequenced (Ogawa et al., 1987). The enzyme is a tetramer of four identical 292 amino acid subunits. In 1984, it was shown that GNMT was identical to a folate binding protein in rat liver cytosol that contained tightly, but not covalently, bound 5-methyltetrahydrofolate pentaglutamate (Cook $\&$ Wagner, 1984). Although the folate ligand does not participate in the methyl transfer reaction, it was shown that it acts as an allosteric inhibitor (Wagner et al., 1985). This property places GNMT at the center of a metabolic mechanism that balances the de novo synthesis of methyl groups via the folate family of coen-

Reprint requests to: R. Pattanayek, Department of Biochemistry, Vanderbilt University School of Medicine, Nashville, Tennessee 37232-0146; e-mail: pattanr@ctrvax.Vanderbilt.edu.

Abbreviations: GNMT, glycine N-methyltransferase; AdoMet, S-adenosylmethionine; AdoHcy, S-adenosylhomocysteine; RMS, root mean square; EDTA, ethylenediaminetetraacetic acid; FPLC, fast protein liquid chromatography; DTT, dithiothreitol. zymes to the availability of pre-formed methionine methyl groups (Balaghi \& Wagner, 1995). Recently, evidence has been provided that a form of GNMT is identical to the " $4 S$ " receptor for polyaromatic hydrocarbons such as benz(a)pyrene (Raha et al., 1994).

The crystal structure of recombinant rat liver GNMT was recently published by Fu et al. (1996). Once the structure of GNMT was traced a significant residual electron density in the region of the active site was identified as AdoMet, one of the substrates. GNMT had apparently been purified and crystallized with AdoMet bound to it. It was also noted that an additional residual electron density peak in the active site was due to the presence of acetate, a component of the solution in which the crystals were grown. Acetate is a competitive inhibitor of glycine (Konishi \& Fujioka, 1987) and so its presence in the crystal structure allowed the placement of the glycine binding site. From this holo GNMT structure (complexed with AdoMet and acetate), it was observed that $\mathrm{N}$-terminal region of each subunit appears as a cork to the entrance of the adjoining subunit. It was predicted that no cork would be present in the apo (free) enzyme to allow the binding of AdoMet. We now report the structure of apo-GNMT in the absence of substrates and the difference between the holo and apo-GNMT structures will be discussed.

\section{Results}

In order to define the conformational changes induced by the cofactor binding to GNMT, the structure of the apo enzyme was 
determined. A ribbon representation of the apo-GNMT tetramer (in stereo) is shown in Figure 1A. GNMT is the only tetrameric member of a family of methyltransferases (Gomi et al., 1992). Each monomer is roughly spherical in shape except for the $24 \mathrm{~N}$-terminal residues. The tetramer can be described as a dimer of dimers. Monomers of the dimer are related by a noncrystallographic twofold rotation. This twofold axis makes a $90^{\circ}$ angle with the crystallographic twofold axis. The dimers of the tetramer are related by crystallographic twofold symmetry. A large solvent channel $(50 \times$ $15 \AA$ ) is located at the center of the tetramer. A stereo view of the monomer is shown in Figure 1B. A monomer is comprised of 292 amino acids and contains 9 alpha helices and 11 beta strands organized into 3 domains. The $\mathrm{N}$-terminal domain contains residues 1-36, the middle domain (designated by Fu et al., 1996, as the S domain) contains residues 176-242 and the C-terminal domain contains residues $37-175$ and 243-292. Residues 1-23 resemble an arm with the elbow bent at $45^{\circ}$, projected away from the main body of the monomer (Fig. 1B). As shown in Figure 1A, the $\mathrm{N}$-terminal arm from the A monomer reaches the diagonally opposite $\mathrm{C}$ monomer via the $\mathrm{B}$ monomer. The elbow part of the arm (residues 8-23) of the A monomer is positioned at the entrance of the active site of the B monomer. Residues 1-7 of the A monomer make anti-parallel $\beta$-strand contacts with the same residues of the D monomer. Similarly, the N-terminal seven residues of the $\mathrm{B}$ monomer make anti-parallel $\beta$-strand contacts with the $\mathrm{N}$-terminal seven residues of the $\mathrm{C}$ monomer. The middle-domain ( $\mathrm{S}$ ) consists of an alpha helix, a large loop, and three anti-parallel beta strands. The $\mathrm{C}$ domain consists of a typical $\alpha / \beta$ Rossmann fold. Hydrophilic residues line the surface and central channel. A large number of aromatic hydrophobic residues lie near and around the active site.

A GNMT dimer occupies the asymmetric unit of the crystal and the RMS difference between the monomers of the dimer is $0.354 \AA$ ( $\mathrm{C}_{\alpha}$ atoms). A correlation between the calculated and observed electron densities for A and B monomers was calculated. With the exception of the loop regions 37-40 and 124-128 and the amino terminus, the correlation is good. The average temperature factor is $33.24 \AA^{2}$ for all atoms $(4,670)$. The $6 \mathrm{~N}$-terminal residues are relatively mobile as indicated by the high temperature factors (50-70 $\AA^{2}$ ). The interface between the dimers, linked by six salt bridges and two hydrogen bonds, has been described in detail by Fu et al. (1996). Hydrogen bonds provide the intermolecular contacts by interlocking $\mathrm{N}$-terminal regions that form the central part of the tetramer.
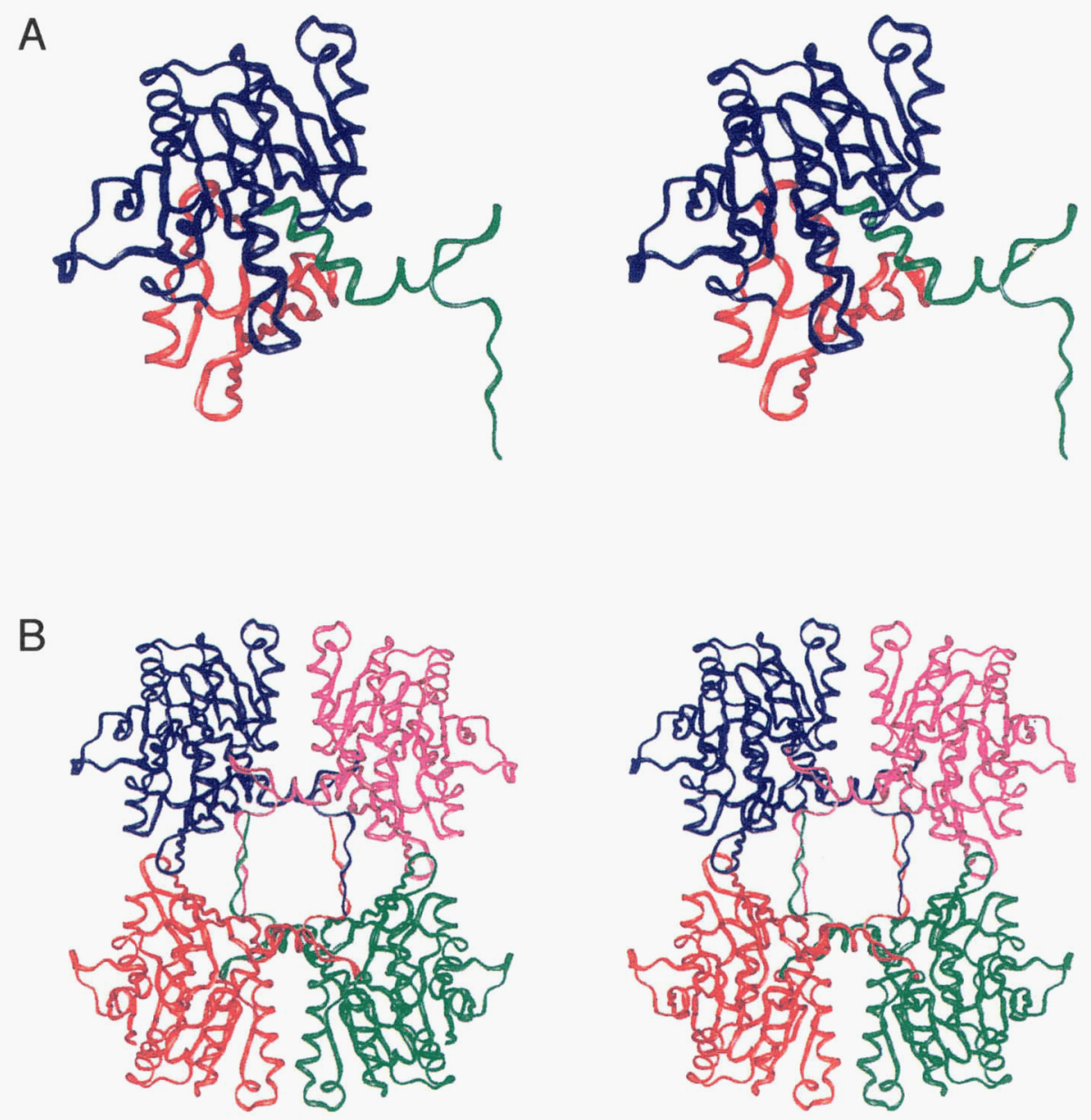

Fig. 1. A: A ribbon representation (stereo) of the GNMT tetramer. The monomers are A (pink), B (blue), C (red), and D (green). The $\mathrm{N}$-terminus of the A monomer reaches the diagonally opposite monomer $\mathrm{C}$ via the $\mathrm{B}$ monomer. B: A ribbon representation (stereo) of monomer B in the same orientation as in Figure 1A. The N-terminus (1-36) is shown in green, the S-domain (176-242) in red, and C-domain (37-175 and 243-292) in blue. 
When apo-GNMT is compared with the structure of GNMT determined with AdoMet and acetate, no large conformational changes are apparent. The apo structure has only a total of $281 \AA^{2}$ more accessible surface area than the holo-structure. This represents only an increase of $1.8 \%$ in total accessible surface area. The RMS deviation for the $584 \mathrm{C}_{\alpha}$ atoms (dimer) of the apo enzyme and the holo (AdoMet/acetate) GNMT structure is $0.575 \AA$ after superimposition of the latter structure onto the structure of apoGNMT. Figure 2 shows the RMS difference of equivalent $C_{\alpha}$ atoms between the two structures and that difference for the $\mathrm{A}$ and $B$ monomers of apo-GNMT. The residue number is shown along the $X$-axis and RMS differences of $\mathrm{C}_{\alpha}$ atoms along the $Y$-axis in $\AA$. Residues that show significant differences (above $1.25 \AA$ ) in the holo vs. apo structures are residues $16,38,39,187$ to 191 (in A monomer), and 187 to 191 (in B monomer). However, because the electron density for amino acids 37 to 40 is poor in the apo model, and these main-chain structures differ in the two monomers, the RMS difference between the holo and apo structures is not likely to be significant for this region. (In the holo structure, the RMS difference of A and B monomer for this loop region $36-39$ is also high.)

GNMT was crystallized in the absence of exogenous AdoMet. However, Fu et al. reported that AdoMet co-purified with GNMT and was clearly present in the electron density map despite the fact that the cofactor was not added to crystallization experiments. Consequently, it was necessary to confirm that our crystals were indeed AdoMet free. The electron density map revealed no potential AdoMet density. A stereo view of the simulated annealing $\left(1,000^{\circ} \mathrm{C}\right)$ omit map $\left(\left|F_{o}\right|-\left|F_{c}\right|\right)$ of the AdoMet binding site is shown in Figure 3 . All atoms within $5 \AA$ from the AdoMet binding site were omitted and SA refinement was done. The density is shown at a $2.5 \sigma$ contour level. The dots represent the positions of the water molecules. The AdoMet molecule (after least-squares fitting of the two structures) has been superimposed on the electron density map to show that there is no density that could correspond to AdoMet. It is important to note that AdoMet density was seen in the complex structure (Fu et al., 1996) at a $2.5 \sigma$ contour level.

Furthermore, no AdoMet could be extracted from the protein set up in crystallization experiments. To confirm the absence of

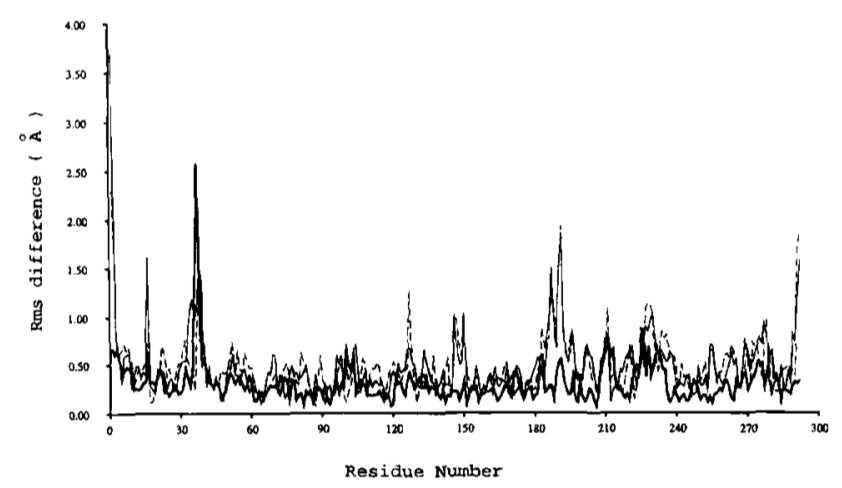

Fig. 2. The RMS differences $(\AA)$ of the $C_{\alpha}$ atoms as a function of residue number. The thick line represents the RMS difference between $A$ and $B$ monomers of apo-GNMT, the thin line corresponds to the RMS difference between the A monomers of apo and AdoMet-GNMT, and the dashed line represents the RMS difference between the B-monomers of apo and AdoMetGNMT. The AdoMet-GNMT structure was least-squares fitted onto apoGNMT before RMS difference calculations.
AdoMet, as deduced from the lack of appropriate electron density, a protein sample from the crystallization experiments, containing $20 \mu \mathrm{g}$ of GNMT, was analyzed by a fluorescence method developed for measuring AdoMet in human plasma. No AdoMet was detected in an aliquot equivalent to 150 pmoles of monomeric GNMT. The method used to detect AdoMet is sensitive to 1 pmole.

\section{Discussion}

The structures of seven methyltransferases are available at present. All of the enzymes catalyze the transfer of a methyl group from AdoMet to a specific substrate. In general, in spite of their low sequence homology, these structures reveal a common $\alpha / \beta$ Rossmann fold that forms the AdoMet binding domain. However, the binding of AdoMet by GNMT contains an additional feature in the form of a unique $S$-domain. Fu et al. (1996) has discussed this in detail.

GNMT plays a central role in the regulation of methyl group metabolism. It is inhibited by 5-methyltetrahydrofolate pentaglutamate as part of a mechanism that relates the de novo synthesis of methyl groups via the family of folate coenzymes to the availability of pre-formed methyl groups (methionine) in the diet. The kinetics of the reaction catalyzed by GNMT are complex. Although normal Michaelis-Menten kinetics are seen with respect to one substrate, glycine, sigmoidal kinetics are seen with respect to AdoMet (Ogawa \& Fujioka, 1982). This is usually interpreted as indicating that the enzyme undergoes a ligand induced conformational change so that the binding of successive molecules of substrate occurs more easily than binding of the first molecule. Such a mechanism is consistent with the fact that GNMT is the only methyltransferase that is tetrameric; the others being either monomeric or dimeric (Gomi et al., 1992). Konishi and Fujioka (1988) showed that tryptic digestion of the native enzyme removes the $\mathrm{N}$-terminal eight amino acids and results in a modified enzyme that is active but has lost its sigmoidal kinetic behavior. This observation clearly indicates that interaction of the subunits is somehow mediated by this eight amino acid segment. In the absence of this segment, the antiparallel beta sheets between monomers $\mathrm{A}$ and $\mathrm{D}$ and between $\mathrm{B}$ and $\mathrm{C}$ are absent, presumably limiting any interaction of the subunits. It is therefore somewhat surprising that the structures of the apo enzyme and the enzyme with AdoMet bound are so similar, as only small, highly localized conformational changes are observed in the binding site.

Some of the important residues at the AdoMet binding site (B monomer) are shown in Figure 4A: apo-GNMT (green), AdoMetGNMT (red), and AdoMet (blue). The relative position of the binding site in the monomer (green ribbon) is shown in Figure 4B, and the orientation of the monomer is close to that of monomer in (Fig. 1B). One side of the AdoMet is covered with the residues 12 to 18 from the neighboring subunit (defined as the "cork" in the holo structure), and these residues superimpose well in both the structures. The other side of the AdoMet is surrounded by the residues 188 to 192 , and these residues were disordered (the temperature factors range from 60 to $86 \AA^{2}$; Fu et al., 1996) in the holo structure. In the apo structure, the electron density for this region is very clear. The side chain of Tyr33B shifts around $1.57 \AA$ in the apo structure. This residue is well determined. There are four water molecules (green) in the apo-GNMT binding site, which were not seen in the AdoMet-GNMT structure. Three of these waters make 

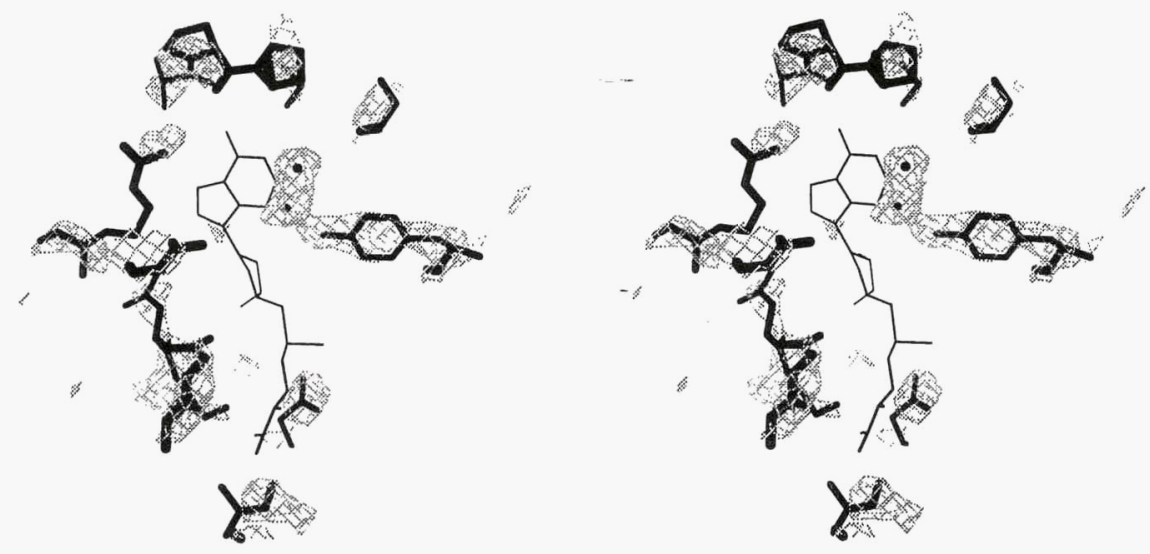

Fig. 3. A stereo view of an omit difference electron density map $\left(\left|F_{o}\right|-\left|F_{c}\right|\right.$, contoured at $\left.2.5 \sigma\right)$ of the apo-GNMT structure surrounding the AdoMet binding site. All atoms within $5 \AA$ from the AdoMet site were omitted and simulated annealing $\left(1,000^{\circ} \mathrm{C}\right)$ refinement was done before the calculation of the omit map. AdoMet is positioned according to the published AdoMet-GNMT structure. Electron density is apparent for the omitted residues. The dots represent the water molecules.

hydrogen bonds with neighboring residues or with other water molecules, and the fourth one is centered between three potential hydrogen bond donors.

Fu et al. speculated that in the absence of AdoMet, the entrance to the active site would be completely open, allowing access of AdoMet to its active site. But, there is no significant difference in the conformation of the proposed "cork" region in the two structures. Residues 12 to 18 from the neighboring subunit superimpose well (Fig. 4). Furthermore, the crystallization conditions for apoGNMT and AdoMet-GNMT are completely different and therefore the lack of conformational changes observed between the two structures is not due to similar crystallization conditions.
A
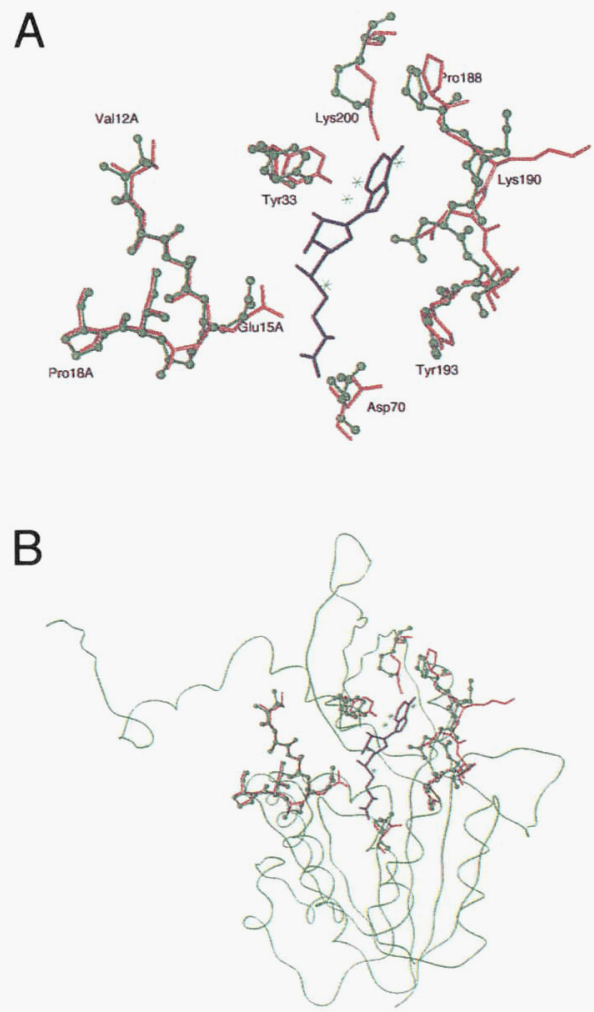
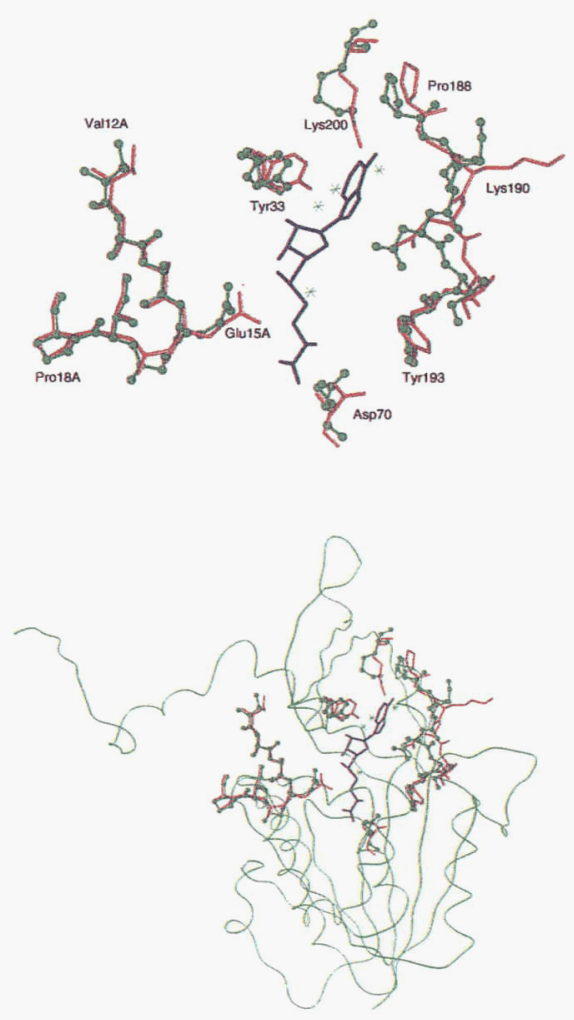

Fig. 4. A: A stereo view of apo-GNMT (green) and AdoMet-GNMT (red) surrounding the AdoMet (blue) binding site in the $\mathrm{B}$ monomer. The waters are shown as crosses. The residues of the cork region from the neighboring A monomer, the loop region residues 188 to 193, Tyr 33, Lys 200, and Asp 70 surrounding the AdoMet binding site are shown. For reference the backbone ribbon of the monomer is included in $\mathbf{B}$. 
Methods

\section{Protein purification and crystallization}

The clone containing the cDNA for rat liver GNMT was a generous gift of Dr. Ogawa. It was transferred to plasmid pRSET and expressed in $E$. coli BL21(DE3) after induction by isopropyl thiogalactoside (IPTG). Cells were lysed using lysozyme followed by sonication. The soluble extract was concentrated using ammonium sulfate by collecting the fraction precipitating between 21 and 31 $\mathrm{g} / \mathrm{mL}$. The pellet was dissolved in $10 \mathrm{mM}$ potassium phosphate ( $\mathrm{pH} 7.0$ ) containing $50 \mathrm{mM} \mathrm{NaCl}, 2 \mathrm{mM}$ EDTA, $10 \mathrm{mM}$ sodium azide, $10 \mathrm{mM}$ 2-mercaptoethanol and $100 \mathrm{mM}$ phenylmethylsulfonyl fluoride and applied to a column of DEAE cellulose (Whatman DE-52) equilibrated with the same buffer. GNMT was present in the flow through and this was applied to a column of Sephacryl S-200 (Whatman). The activity was present as a single peak. Final purification was carried out by FPLC using a Baker QUAT column equilibrated with $50 \mathrm{mM}$ Tris buffer $(\mathrm{pH} 8.8)$ containing $1 \mathrm{mM}$ sodium azide, $5 \mathrm{mM}$ dithiothreitol, and $10 \mathrm{mM}$ 2-mercaptoethanol with a $\mathrm{NaCl}$ gradient. GNMT eluted in a single symmetrical peak. GNMT activity was assayed as described by Wagner et al. (1985).

Purified recombinant protein was used for protein crystallization. Crystals were grown from $0.1 \mathrm{M}$ sodium citrate, $1 \mathrm{M}$ ammonium phosphate, $5 \%$ glycerol and $100 \mathrm{mM} \mathrm{NaCl}, \mathrm{pH} 6.8$ at $11^{\circ} \mathrm{C}$ by hanging drop vapor diffusion. Protein concentration was approximately $8 \mathrm{mg} / \mathrm{mL}$ (in $10 \mathrm{mM}$ Tris, $\mathrm{pH} 8.3,1 \mathrm{mM} \mathrm{NaN}_{3}$, $10 \mathrm{mM}$ beta mercaptoethanol, $2 \mathrm{mM}$ DTT, and $2 \mathrm{mM}$ EDTA). Equal volumes of protein solution and well solution were used to set up the $5 \mu \mathrm{L}$ drops. It takes four to five weeks to grow crystals large enough to produce high quality $\mathrm{X}$-ray data.

\section{$X$-ray data collection and processing}

The crystals are not stable during data collection at room temperature. However, the crystal life time is significantly improved in the $\mathrm{X}$-ray beam by freezing the crystal at $-165^{\circ} \mathrm{C}$. The $\mathrm{X}$-ray source was $\mathrm{Cu}_{\alpha}$ radiation from a Rigaku RU-200 rotating anode generator. The diffraction data, collected on a $R$-axis II image plate system, were processed using the program DENZO (Otwinowski, 1993; Minor, 1993). The data were $93.8 \%$ complete in the resolution range 50 to $2.5 \AA$ (254,910 total reflections, 22,481 unique reflections, $R_{\text {merge }}=10.6 \%, I / \sigma=10.6$ ) (Table 1A). These crystals belong to the orthorhombic space group $\mathrm{P} 2{ }_{1} 2{ }_{1} 2(a=85.39, b=$

Table 1A. Summary of $X$-ray data collection statistics

\begin{tabular}{lc}
\hline Wavelength $(\AA)$ & 1.54 \\
Temperature $\left({ }^{\circ} \mathrm{C}\right)$ & -165 \\
Resolution $(\AA)$ & 2.5 \\
Total observations & 254,910 \\
Unique reflections & 22,481 \\
Completeness of data $(\%)$ & $93.8(87.0)^{\mathrm{a}}$ \\
Intensity $(I / \sigma)$ & $10.59(2.73)$ \\
$R_{\text {merge }}(\%)$ & $10.6 \quad(41.7)^{\mathrm{a}}$ \\
\hline
\end{tabular}

${ }^{a}$ The numbers in the parentheses indicate values in the outer resolution shell $(2.54$ to 2.50$)$.

${ }^{\mathrm{b}} R_{\text {merge }}=\sum|I-\langle I\rangle| / \sum I$, where $I$ is the intensity measurement for a reflection and $\langle I\rangle$ is the mean value for this reflection.
174.21, $c=44.71 \AA$ ) and diffract to at least $2.2 \AA$ resolution (data were processed to $2.5 \AA$ ). An asymmetric unit is occupied by two monomers.

\section{Structure solution and refinement}

Although the crystallization conditions $(0.1 \mathrm{M}$ sodium citrate, $1 \mathrm{M}$ ammonium phosphate, $5 \%$ glycerol and $100 \mathrm{mM} \mathrm{NaCl}, \mathrm{pH} 6.8$ ) differ significantly from those described by $\mathrm{Fu}$ et al. (1996) (100 $\mathrm{mM}$ ammonium acetate, $50 \mathrm{mM} \mathrm{NaCl}$, and 13\% (w/v) PEG $4000, \mathrm{pH}=5.6$ ), the crystals were isomorphous. Rigid body refinement of the structure reported by Fu et al. (1xva.pdb) without water and ligands but with our data (where $F>2 \sigma$ ) provided the initial phases $\left(R_{\text {crystal }}=38.3 \%\right.$ ) for our model. Ten percent of the data were set aside at the beginning of the refinement to monitor $R_{\text {free }}$. The temperature factors for all the atoms were set to $15.0 \AA^{2}$. After 50 cycles of positional refinement using XPLOR (Brünger, 1993 ) the first $\left|F_{o}\right|-\left|F_{c}\right|$ electron density map was calculated. This map did not show any electron density that could be interpreted as AdoMet. Simulated annealing (SA), positional and temperature factor refinements were done using XPLOR (Brünger et al., 1990). In the initial stages of the refinement, NCS restraints were applied to the dimer subunits and these restraints were released at the later stages of the refinement. The model was rebuilt where necessary using the program $O$ (Jones et al., 1991). One hundred five water molecules were located with the help of the difference Fourier map. After the addition of solvent molecules, a cycle of bulk solvent refinement was performed using XPLOR. A simulated annealing omit (all atoms within $5 \AA$ from the AdoMet binding site were omitted) map $\left(\left|F_{o}\right|-\left|F_{c}\right|\right)$ was calculated and did not show any additional density that may be attributed to AdoMet. The quality of the model was assessed by PROCHECK (Laskowski et al., 1993). There are no outliers in the Ramachandran Plot $(80 \%$ in the most favored regions and $20 \%$ in additional allowed region). A final difference Fourier map $\left(\left|F_{o}\right|-\left|F_{c}\right|\right)$ was calculated, and no significant electron density was observed that was unaccounted for. The final refinement statistics are given in Table 1B

To confirm the absence of AdoMet, as deduced from the lack of appropriate electron density, a protein sample from the crystallization experiments, containing $20 \mu \mathrm{g}$ of GNMT, was analyzed by a fluorescence method developed for measuring AdoMet in human plasma. Trichloroacetic acid was added to a final concentration of $10 \%$ in order to denature the protein. The trichloroacetic acid was then removed by extraction with trioctylamine and 1,1,3trichlorotrifluoroethane. An aliquot equivalent to 150 pmoles of

Table 1B. Refinement statistics

\begin{tabular}{lc}
\hline$R_{\text {crystal }}{ }^{a}(\%)$ & 21.9 \\
$R_{\text {free }}{ }^{(\%)}$ & 31.8 \\
RMS deviations in bond length $(\AA)$ & 0.007 \\
RMS deviations in bond angle $\left(^{\circ}\right)$ & 1.325 \\
RMS deviations in torsion angle $\left(^{\circ}\right)$ & 27.776 \\
Average $B$-values $\left(\AA^{2}\right)$ & 33.24
\end{tabular}

${ }^{a} R=\sum\left|F_{o}-F_{c}\right| / \sum\left|F_{o}\right|$, where $F_{o}$ and $F_{c}$ are the observed and calculated structure factor amplitudes, respectively, and RMS is the root mean square. 
GNMT monomer was then derivatized with naphthalene dialdehyde to form the fluorescent isoindole and subsequently chromatographed on a C-18 column. The fluorescence of the eluate was monitored (excitation at $420 \mathrm{~nm}$; emission at $483 \mathrm{~nm}$ ). There was no evidence for the presence of AdoMet. This method is sensitive to 1 pmole.

\section{Acknowledgments}

This work was supported by National Institute of Health grants DK46788 and DK15289 and the Department of Veterans Affairs to C.W., National Institutes of Health grant GM55420 to M.E.N. and grant DK26657 to Clinical Nutrition Research Unit, Vanderbilt University School of Medicine.

\section{References}

Balaghi M, Wagner C. 1995. Folate deficiency inhibits pancreatic amylase secretion in rats. Am J Clin Nutr 61(1):90-96.

Brünger AT, Krukowski A, Erickson JW. 1990. Slow cooling protocols for crystallographic refinement by simulated annealing. Acta Cryst A 46:585-593.

Brünger AT. 1993. X-PLOR 3.I: A system for X-ray crystallography and NMR. New Haven, CT: Yale University Press.

Cook RJ, Wagner C. 1984. Glycine N-methyltransferase is a folate binding protein of rat liver cytosol. Proc Natl Acad Sci USA 81(12):3631-3634.

Fu Z, Hu Y. Konishi K, Takata Y, Ogawa H, Gomi T, Fujioka M, Takusagawa F. 1996. Crystal structure of glycine N-methyltransferase from rat liver Biochemistry 35(37):11985-11993.

Gomi T, Tanihara K, Date T, Fujioka M. 1992. Rat guanidoacetate methyltrans- ferase: Mutation of amino acids within a common sequence motif of mammalian methyltransferase does not affect catalytic activity but alters proteolytic susceptibility. Int J Biochem 24:1639-1649.

Heady JE, Kerr SJ. 1973. Purification and characterization of glycine N-methyltransferase. J Biol Chem 248(1):69-72.

Jones TA, Zou JY, Cowan SW, Kjeldgaard M. 1991. Improved methods for building protein models in electron density maps and location of errors in these models. Acta Cryst A47:1 10-119.

Konishi K, Fujioka M. 1987. Chemical modification of a functional arginine residue of rat liver glycine methyltransferase. Biochemistry 26(25):84968502.

Konishi K, Fujioka M. 1988. Rat liver glycine methyltransferase. J Biol Chem 264:13381-13385.

Laskowski RA, MacArthur MW, Moss DS, Thornton M. 1993. PROCHECK: A program to check the stereochemical quality of protein structures. $J \mathrm{Appl}$ Crystallogr 26:946-950.

Minor W. 1993. XDISPLAYF program. West Lafayette: Purdue University.

Ogawa H, Fujioka M. 1982. Purification and properties of glycine $\mathrm{N}$-methyltransferase from rat liver. $J$ Biol Chem 257(7):3447-3452.

Ogawa H, Konishi K, Takata Y, Nakashima H, Fujioka M. 1987. Rat glycine methyltransferase. Complete amino acid sequence deduced from a cDNA clone and characterization of the genomic DNA. Eur J Biochem 168(1):141151 .

Otwinowski Z. 1993. DENZO: An oscillation data processing program for macromolecular crvstallography. New Haven, Connecticut: Yale University.

Raha A, Wagner C, MacDonald RG, Bresnick E. 1994. Rat liver cytosolic 4 S polycyclic aromatic hydrocarbon-binding protein is glycine $\mathrm{N}$-methyltransferase. $J$ Biol Chem 269(8):5750-5756.

Wagner C, Briggs WT, Cook RJ. 1985. Inhibition of glycine N-methyltransferase activity by folate derivatives: Implications for regulation of methyl group metabolism. Biochem Biophys Res Commun 127(3):746-752. 